\title{
A Generalized Preconditioned MPHSS Method for a Class of Complex Symmetric Linear System
}

\author{
Zhongshe Gao \\ School of Mathematics and Statistics \\ Tianshui Normal University \\ Tianshui, 741001, P. R. of China \\ e-mail: gaozhongshe@hotmail.com
}

\begin{abstract}
In this paper, with the MHSS and preconditioned MHSS methods, we get a generalized preconditioned MHSS method with a kind of complex symmetric linear systems. This method is a two-parameter iteration process, which can optimize the iterative process. The sequence of iterative produced by the generalized preconditioned MHSS method is proved to be convergence to the unique solution of the complex symmetric linear system.
\end{abstract}

Keywords- generalized preconditioned MHSS; complex symmetric linear systems; iterative methods; iteration matrix.

\section{INTRODUCTION}

In many scientific computing and engineering applications, we need solve the complex symmetric linear systems, such as structural dynamics, diffusing optical tomography, lattice quantum chromodynamics and so on. So we consider the iterative solution of systems of complex symmetric linear equations

$$
A x=b, \quad A \in C^{n \times n} \text { and } x, b \in C^{n},
$$
form

where $A \in C^{n \times n}$ is a complex symmetric matrix of the

$$
A=W+i T,
$$

and $W, T \in C^{n \times n}$ are real symmetric matrices, with $W$ being positive definite and $T$ postive semidefinite. Here and in the sequel we use $i=\sqrt{-1}$ to denote the imaginary unit. We assume $T \neq 0$, which imply that $A$ is non-Hermitian.

The Hermitian and skew-Hermitian parts of the complex symmetric matrix $A \in C^{n \times n}$ are given by

$$
H=\frac{1}{2}\left(A+A^{*}\right)=W \text { and } S=\frac{1}{2}\left(A-A^{*}\right)=i T,
$$

where $A \in C^{n \times n}$ is a non-Hermitian, but positive definite matrix, $A^{*}$ is used to denote the conjugate transpose of the matrix $A$. Based on the Hermitian and skew-Hermitian splitting(HSS).

$$
A=H+S \text {. }
$$

Bai, et al $[1,3,4]$ proposed iterative methods called HSS and preconditioned HSS methods based on this kind of particular matrix splitting, in reference[2], Bai, et al proposed MHSS methods for a class of complex symmetric linear system, which based on one postive parameter, and in reference[5], Yang, et al proposed GPHSS methods for a non-hermitian postive definete linear system, which based on two postive parameters, in this paper, we will propose GPMHSS methods for a class of complex symmetric linear system, which depend on two postive parametera.

The paper is organized as follows. In Section II, the GPMHSS method is given. In Section III, the convergence properties of the GPMHSS method is discussed. Finally, we offer a brief conclusion. For the upper bound of the spectral radius of the iteration matrix, the optimal parameters with GPMHSS method will be discussed in the following paper.

\section{THE GPMHSS ITERATION METHOD}

By making use of the special structure of the coefficient matrix $A \in C^{n \times n}$ of the complex symmetric linear system (1), in this section, we derive a general of the PMHSS iteration method, which based on the GPHSS method ([5]) and MHSS method ([2]). We produce from the one parameter in the iterated procedure in reference [2], we will introduce two different parameters $\alpha$ and $\beta$ in the following. This lead to the generalized PMHSS Iteration method.

Method(The Generalized PMHSS Iteration Method) Let $x^{(0)} \in C^{n}$ be an arbitrary initial guess, For $k=0,1,2, \cdots$ until the sequence of the iterates $\left\{x^{(k)}\right\}_{k=1}^{\infty} \subset C^{n}$ converges, compute the next iterate $x^{(k+1)}$ according to the following procedure

$$
\left\{\begin{array}{l}
(\alpha P+W) x^{\left(k+\frac{1}{2}\right)}=(\alpha P-i T) x^{(k)}+b, \\
(\beta P+T) x^{\left(k+\frac{1}{2}\right)}=(\beta P-i W) x^{(k)}-i b .
\end{array}\right.
$$

Where $\alpha$ is a given nonnegative constant and $\beta$ a given positive constant, and $P$ is anHermitian positive definite matrix. 


\section{CONVERGENCE ANALYSIS}

Lemma $^{[5]}$ Let $A \in C^{n \times n}, A=M_{i}-N_{i}(i=1,2)$ be two splitting of matrix $A$, and let $x^{(0)} \in C^{n}$ as a given initial vector. If $x^{(k)}$ is iteration sequence of two-step defined by

$$
\left\{\begin{array}{l}
M_{1} x^{\left(k+\frac{1}{2}\right)}=N_{1} x^{(k)}+b, \\
M_{2} x^{(k+1)}=N_{2} x^{\left(k+\frac{1}{2}\right)}-i b, \quad k=0,1,2, \cdots
\end{array}\right.
$$

then

$$
x^{(k+1)}=M_{2}^{-1} N_{2} M_{1}^{-1} N_{1} x^{(k)}+M_{2}^{-1}\left(N_{2} M_{1}^{-1}-i I\right) b,
$$

Furthermore, if $\rho\left(M_{2}^{-1} N_{2} M_{1}^{-1} N_{1}\right)<1$, the iterative sequence $\left\{x^{(k)}\right\}$ convergence to the unique $x^{*}$ of the system (1) for any initial $x^{(0)}$.

Theorem Let $A \in C^{n \times n}$ is a positive definite matrix, $P \in C^{n \times n}$ is an Hermitian definite matrix, $\Lambda_{1}$ and $\Lambda_{2}$ be spectral sets of matrix $P^{-1} W$ and matrix $P^{-1} T$, respectively, let

$$
\begin{array}{ll}
\lambda_{\text {max }}=\max _{\lambda_{k} \in \Lambda_{1}}\left\{\lambda_{k}\right\}, & \lambda_{\text {min }}=\min _{\lambda_{k} \in \Lambda_{1}}\left\{\lambda_{k}\right\} ; \\
\mu_{\text {max }}=\max _{\mu_{k} \in \Lambda_{2}}\left\{\mu_{k}\right\}, & \left.\mu_{\text {min }}=\min _{\mu_{k} \in \Lambda_{2}}\right\} .
\end{array}
$$

Then, the GMPHSS iteration $x^{(k)}$ converges to the unique solution $x^{*} \in C^{n}$ of the linear system (1) if the parameters $\alpha$ and $\beta$ satisfy

$$
(\alpha, \beta) \in \bigcup_{i=1}^{4} \Omega_{i},
$$

Where

$$
\begin{aligned}
& \Omega_{1}=\left\{(\alpha, \beta) \mid \beta \leq \alpha<\alpha^{*}(\beta), \varphi_{1}(\alpha, \beta)<0\right\}, \\
& \Omega_{2}=\left\{(\alpha, \beta) \mid \alpha<\min \left\{\beta, \alpha^{*}(\beta)\right\}, \varphi_{2}(\alpha, \beta)<0\right\}, \\
& \Omega_{3}=\left\{(\alpha, \beta) \mid \alpha^{*}(\beta) \leq \alpha<\beta, \varphi_{3}(\alpha, \beta)<0\right\}, \\
& \Omega_{4}=\left\{(\alpha, \beta) \mid \alpha \geq \max \left\{\beta, \alpha^{*}(\beta)\right\}, \varphi_{4}(\alpha, \beta)<0\right\} . \\
& \quad \text { with functions } \varphi_{i}(\alpha, \beta)(i=1,2,3,4) \quad \text { and } \\
& \alpha^{*}(\beta) \text { denoted by }
\end{aligned}
$$

$$
\begin{aligned}
& \varphi_{1}(\alpha, \beta)=\left(\lambda_{\min }^{2}-\mu_{\min }\left(2 \beta+\mu_{\min }\right)\right) \alpha^{2}-2\left(\lambda_{\min } \beta^{2}\right. \\
&\left.+\lambda_{\min } \mu_{\min }^{2}-\left(\beta-\lambda_{\min }\right)^{2} \mu_{\min }\right) \alpha \\
&+\beta\left(\beta\left(\mu_{\min }^{2}-\lambda_{\min }^{2}\right)-2 \lambda_{\min }^{2} \mu_{\min }\right)<0 ; \\
& \varphi_{2}(\alpha, \beta)=\left(\lambda_{\min }^{2}-\mu_{\max }\left(2 \beta+\mu_{\max }\right) \alpha^{2}-2\left(\lambda_{\min } \beta^{2}\right.\right. \\
&\left.+\lambda_{\min } \mu_{\max }^{2}-\left(\beta-\lambda_{\min }\right)^{2} \mu_{\max }\right) \alpha \\
&+\beta\left(\beta\left(\mu_{\max }^{2}-\lambda_{\min }^{2}\right)-2 \lambda_{\min }^{2} \mu_{\max }\right)<0 ; \\
& \varphi_{3}(\alpha, \beta)=\left(\lambda_{\max }^{2}-\mu_{\max }\left(2 \beta+\mu_{\max }\right)\right) \alpha^{2}-2\left(\lambda_{\max } \beta^{2}\right. \\
&\left.+\lambda_{\max } \mu_{\max }^{2}-\left(\beta-\lambda_{\max }\right)^{2} \mu_{\max }\right) \alpha \\
&+\beta\left(\beta\left(\mu_{\max }^{2}-\lambda_{\max }^{2}\right)-2 \lambda_{\max }^{2} \mu_{\max }\right)<0 ; \\
& \varphi_{4}(\alpha, \beta)=\left(\lambda_{\max }^{2}-\mu_{\min }\left(2 \beta+\mu_{\min }\right)\right) \alpha^{2}-2\left(\lambda_{\max } \beta^{2}\right. \\
&\left.+\lambda_{\max } \mu_{\min }^{2}-\left(\beta-\lambda_{\max }\right)^{2} \mu_{\min }\right) \alpha \\
&+\beta\left(\beta\left(\mu_{\min }^{2}-\lambda_{\max }^{2}\right)-2 \lambda_{\max }^{2} \mu_{\min }\right)<0 ; \\
& \beta^{2}-\lambda_{\max } \lambda_{\min }+\sqrt{\left(\beta^{2}+\lambda_{\max }^{2}\right)\left(\beta^{2}+\lambda_{\min }^{2}\right)} \\
& \lambda_{\min }+\lambda_{\max }
\end{aligned}
$$

where $\alpha^{*}(\beta) \in\left[\lambda_{\text {min }}, \lambda_{\text {max }}\right]$, the intersection point of $\alpha^{*}(\beta)$ and $\alpha=\beta$ is $\beta_{0}$, where $\beta_{0}=\sqrt{\lambda_{\min } \lambda_{\max }}$.

Proof By putting $M_{1}=\alpha P+W, M_{2}=\beta P+T$, $N_{1}=\alpha P-i T, N_{2}=\beta P-i W$, in the lemma and noting that $\alpha P+W$ and $\beta P+T$ are nonsingular for any nonnegative constant $\alpha$ and positive $\beta$, one can obtain the iteration matrix of GMPHSS method as follow $M(\alpha, \beta)=(\beta P+T)^{-1}(\beta P-i W)(\alpha P+W)^{-1}(\alpha P-i T)$.

The spectral radius of the iteration matrix satisfies clearly

$$
\begin{aligned}
& \rho(M(\alpha, \beta)) \\
& =\rho\left((\beta P+T)^{-1}(\beta P-i W)(\alpha P+W)^{-1}(\alpha P-i T)\right) \\
& =\rho\left((\beta P-i W)(\alpha P+W)^{-1}(\alpha P-i T)(\beta P+T)^{-1}\right) \\
& \leq\left\|(\beta P-i W)(\alpha P+W)^{-1}(\alpha P-i T)(\beta P+T)^{-1}\right\| \\
& \leq\left\|(\beta P-i W)(\alpha P+W)^{-1}\right\|_{2}\left\|(\alpha P-i T)(\beta P+T)^{-1}\right\|_{2}
\end{aligned}
$$

Because $W \in R^{n \times n}$ and $T \in R^{n \times n}$ are symmetric, there exits orthogonal matrices $U, V \in R^{n \times n}$ such that

$$
U^{T} W U=\Lambda_{W}, V^{T} W V=\Lambda_{T},
$$

where $\Lambda_{W}=\operatorname{diag}\left(\lambda_{1}, \lambda_{2}, \cdots, \lambda_{n}\right)$,

$$
\Lambda_{T}=\operatorname{diag}\left(\mu_{1}, \mu_{2}, \cdots, \mu_{n}\right)
$$

with $\lambda_{i}, \mu_{i}(i=1,2, \cdots, n)$ being the eigenvalues of the matrices $W$ and $T$, respectively, by assumption it holds that $\lambda_{i} \geq 0$ and $\mu_{i} \geq 0$, for the orthogonal invariance of the 
Euclid norm $\|\cdot\|_{2}$, we can obtain the following upper bound on $\rho(M(\alpha, \beta))$

$$
\begin{aligned}
& \rho(M(\alpha, \beta)) \\
& \leq\left\|(\beta P-i W)(\alpha P+W)^{-1}\right\|_{2}\left\|(\alpha P-i T)(\beta P+T)^{-1}\right\|_{2} \\
& \leq\left\|\left(\beta P-i \Lambda_{W}\right)\left(\alpha P+\Lambda_{W}\right)^{-1}\right\|_{2}\left\|\left(\alpha P-i \Lambda_{T}\right)\left(\beta P+\Lambda_{T}\right)^{-1}\right\|_{2} \\
& =\max _{\lambda_{j} \in s p\left(P^{-1} W\right)}\left|\frac{\beta-i \lambda_{j}}{\alpha+\lambda_{j}}\right| \cdot \max _{\mu_{j} \in \operatorname{sp}\left(P^{-1} T\right)}\left|\frac{\alpha-i \mu_{j}}{\beta+\mu_{j}}\right| \\
& =\max _{\lambda_{j} \in \operatorname{sp}\left(P^{-1} W\right)} \frac{\sqrt{\beta^{2}+\lambda_{j}^{2}}}{\alpha+\lambda_{j}} \cdot \max _{\mu_{j} \in \operatorname{sp}\left(P^{-1} T\right)} \frac{\sqrt{\alpha^{2}+\mu_{j}^{2}}}{\beta+\mu_{j}} \\
& \leq \max _{\lambda_{j} \in \sin \left(P^{-1} W\right)} \frac{\sqrt{\beta^{2}+\lambda_{j}^{2}}}{\alpha+\lambda_{j}} \cdot \max _{\mu_{j} \in \operatorname{sp}\left(P^{-1} T\right)} \frac{\alpha+\mu_{j}}{\beta+\mu_{j}}
\end{aligned}
$$

Since $\alpha \geq 0, \beta>0$, it follows that hence using

$\alpha^{*}(\beta)=\frac{\beta^{2}-\lambda_{\text {max }} \lambda_{\text {min }}+\sqrt{\left(\beta^{2}+\lambda_{\text {max }}^{2}\right)\left(\beta^{2}+\lambda_{\min }^{2}\right)}}{\lambda_{\text {min }}+\lambda_{\text {max }}}$,

and we also have

$$
\max _{\lambda_{j} \in S p\left(P^{-1} W\right)} \frac{\sqrt{\beta^{2}+\lambda_{j}^{2}}}{\alpha+\lambda_{j}}= \begin{cases}\frac{\sqrt{\beta^{2}+\lambda_{\max }^{2}}}{\alpha+\lambda_{\max }}, & \alpha \geq \alpha^{*}(\beta) ; \\ \frac{\sqrt{\beta^{2}+\lambda_{\min }^{2}}}{\alpha+\lambda_{\min }}, & \alpha<\alpha^{*}(\beta) .\end{cases}
$$

Similarly, we can discuss the matrix $T$.

$$
\max _{\mu_{j} \in S p\left(P^{-1} T\right)} \frac{\alpha+\mu_{j}}{\beta+\mu_{j}}= \begin{cases}\frac{\alpha+\mu_{\min }}{\beta+\mu_{\min }}, & \alpha \geq \beta, \\ \frac{\alpha+\mu_{\max }}{\beta+\mu_{\max }}, & \alpha<\beta .\end{cases}
$$

Now let us divide the region $\Omega=\{(\alpha, \beta) \mid \alpha \geq 0, \beta>0\}$ into four subregion

$$
\begin{aligned}
& \Omega_{1}=\left\{(\alpha, \beta) \mid \beta \leq \alpha<\alpha^{*}(\beta)\right\}, \\
& \Omega_{2}=\left\{(\alpha, \beta) \mid \alpha<\min \left\{\beta, \alpha^{*}(\beta)\right\}\right\}, \\
& \Omega_{3}=\left\{(\alpha, \beta) \mid \alpha^{*}(\beta) \leq \alpha<\beta\right\}, \\
& \Omega_{4}=\left\{(\alpha, \beta) \mid \alpha \geq \max \left\{\beta, \alpha^{*}(\beta)\right\}\right\} .
\end{aligned}
$$

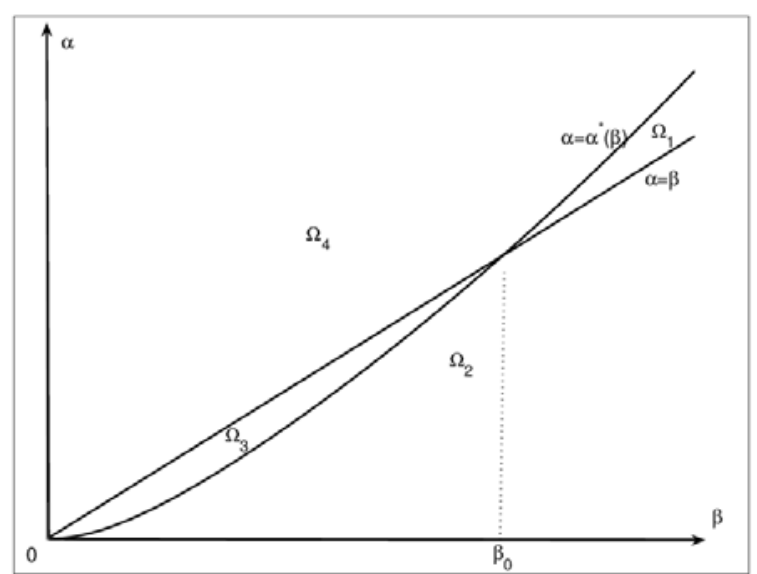

Figure 1. THE FOUR Subregions OF $\Omega$.

(I) For $(\alpha, \beta) \in \Omega_{1}$, we deduce easily from the formula (7-8) following the inequality

$$
\rho(M(\alpha, \beta)) \leq \frac{\sqrt{\beta^{2}+\lambda_{\min }^{2}}}{\alpha+\lambda_{\min }} \cdot \frac{\alpha+\mu_{\min }}{\beta+\mu_{\min }}<1,
$$

if and only if

$$
\begin{aligned}
\varphi_{1}(\alpha, \beta)= & \left(\lambda_{\min }^{2}-\mu_{\min }\left(2 \beta+\mu_{\min }\right)\right) \alpha^{2}-2\left(\lambda_{\min } \beta^{2}\right. \\
& \left.+\lambda_{\min } \mu_{\min }^{2}-\left(\beta-\lambda_{\min }\right)^{2} \mu_{\min }\right) \alpha \\
& +\beta\left(\beta\left(\mu_{\min }^{2}-\lambda_{\min }^{2}\right)-2 \lambda_{\min }^{2} \mu_{\min }\right)<0
\end{aligned}
$$

(II) For $(\alpha, \beta) \in \Omega_{2}$, we deduce easily from the formula (7-8) following the inequality

$$
\begin{aligned}
\rho(M(\alpha, \beta)) & \leq \frac{\sqrt{\beta^{2}+\lambda_{\min }^{2}}}{\alpha+\lambda_{\min }} \cdot \frac{\alpha+\mu_{\max }}{\beta+\mu_{\max }}<1 \Leftrightarrow \\
\varphi_{2}(\alpha, \beta)= & \left(\lambda_{\min }^{2}-\mu_{\max }\left(2 \beta+\mu_{\max }\right)\right) \alpha^{2}-2\left(\lambda_{\min } \beta^{2}\right. \\
& \left.+\lambda_{\min } \mu_{\max }^{2}-\left(\beta-\lambda_{\min }\right)^{2} \mu_{\max }\right) \alpha \\
& +\beta\left(\beta\left(\mu_{\max }^{2}-\lambda_{\min }^{2}\right)-2 \lambda_{\min }^{2} \mu_{\max }\right)<0
\end{aligned}
$$

(III) For $(\alpha, \beta) \in \Omega_{3}$, we deduce easily from the formula (7-8) following the inequality 


$$
\begin{aligned}
\rho(M(\alpha, \beta)) \leq & \frac{\sqrt{\beta^{2}+\lambda_{\max }^{2}}}{\alpha+\lambda_{\max }} \cdot \frac{\alpha+\mu_{\max }}{\beta+\mu_{\max }}<1 \Leftrightarrow \\
\varphi_{3}(\alpha, \beta)= & \left(\lambda_{\max }^{2}-\mu_{\max }\left(2 \beta+\mu_{\max }\right)\right) \alpha^{2}-2\left(\lambda_{\max } \beta^{2}\right. \\
& \left.+\lambda_{\max } \mu_{\max }^{2}-\left(\beta-\lambda_{\max }\right)^{2} \mu_{\max }\right) \alpha \\
& +\beta\left(\beta\left(\mu_{\max }^{2}-\lambda_{\max }^{2}\right)-2 \lambda_{\max }^{2} \mu_{\max }\right)<0 ;
\end{aligned}
$$

(IV) For $(\alpha, \beta) \in \Omega_{4}$, we deduce easily from the formula (7-8) following the inequality. Obviously, the righthand side is less than 1 , if and only if

$$
\begin{aligned}
\varphi_{4}(\alpha, \beta)= & \left(\lambda_{\max }^{2}-\mu_{\min }\left(2 \beta+\mu_{\min }\right)\right) \alpha^{2}-2\left(\lambda_{\max } \beta^{2}\right. \\
& \left.+\lambda_{\max } \mu_{\min }^{2}-\left(\beta-\lambda_{\max }\right)^{2} \mu_{\min }\right) \alpha \\
& +\beta\left(\beta\left(\mu_{\min }^{2}-\lambda_{\max }^{2}\right)-2 \lambda_{\max }^{2} \mu_{\min }\right)<0 ;
\end{aligned}
$$

Therefore, we have obtained $\rho(M(\alpha, \beta))<1$, and for any $(\alpha, \beta) \in \bigcup_{i=1}^{4} \Omega_{i}$, the proof is completed.

\section{CONCLUSION}

As a method for accelerating convergence of iteration for the large sparse non-Hermitian positive definite system of linear equations, we get a two-parameter generalized preconditioned GPHSS method. Obviously, this is a kind of generalization of the classical HSS method because when we take $\alpha=\beta$ without using the preconditioner $P$, we will return to the HSS method. In this paper, we prove that the iterative sequence produced by GPHSS method converge to the unique solution to the system of linear equations, when the parameters $\alpha$ and $\beta$ satisfy moderate conditions which take PHSS method as a special case. The optimal parameters for GPMHSS method will be discussed in the following paper.

\section{ACKNOWLEDGMENT}

This work is partially supported by Project of Education Department in Gansu Province under grant No.1108B-03.

\section{REFERENCES}

[1] Z.-Z. Bai, G.H. Golub and C.-K. Li, Convergence properties of preconditioned Hermitian and skew-Hermitian splitting methods for nonHermitian positive semidefinite matrices, Math. Comput., 76(2007), pp.287-298.

[2] Z.-Z. Bai, M. Benzi and F. Chen, Modified HSS iteration methods for a class of complex symmetric systems linear systems, Computing, 87(2010), pp.93-111.

[3] Z.-Z. Bai, G.H. Golub and M.K. Ng, Hermitian and skewHermitiansplitting methods for non-Hermitian positive definite linear systems, SIAM J. Matrix Analysis Appl., 24(2003), pp. 603-626.

[4] Z.-Z. Bai, G.H. Golub and J.-Y. Pan, Preconditioned Hermitian and skew-Hermitian splitting methods for non-Hermitian positive semidefinite linear systems, Numer. Math., 98(2004), pp. 1-32.

[5] A.-L. Yang, J. An, Y.-J. Wu, A generalized preconditioned HSS method for non-Hermitian positive definite linear systems, Appl. Math. comput., 216(2010), 1715-1722.. 